Bull. Fac. Agric., Cairo Univ., 63: 439-449 (2012).

\title{
DIALLEL ANALYSIS USING HAYMAN METHOD TO STUDY GENETIC ARCHITECTURE OF VEGETATIVE GROWTH IN PEAS (Pisum sativum L.)
}

(Received:29.9.2012)

\author{
By \\ K. B. Esho, M. K. Al-Kumar and J. M. S. Jubreal* \\ Department of Horticulture and Landscaping, College of Agriculture and Forestry, \\ Mosul University and *Scientific Research Center, Faculty of Science, Dohuk University
}

\begin{abstract}
This study was carried out during (2010/2011) growing season in the Vegetative Field Research, Department of Horticulture and Landscape Design, College of Agriculture and Forestry , Mosul University . The aim of this research was to study Diallel Analysis using Hayman Method to investigate genetic architecture of vegetative growth in peas (Pisum sativum L.) . Seven genetic lines were used namely (1=G.S.C.22763, 2=P.S.305301572 , 3= Thomas Laxton , 4= Solara, 5=Pitet Provael, 6= Duna Pea, $7=$ English) . These genotypes were crossed in all possible combinations (Full Diallel Cross) . The seeds of seven parents and their F1's including reciprocals were tested using Randomized Complete Block Design with three replications. The following traits: plant height $(\mathrm{cm})$, number of branches per plant, number of leaves per plant, leave area $(\mathrm{cm})$ leave area index, fresh and dry weight of vegetative growth (g) and the total chlorophyll in leaves were also studied. Results showed that the value of ( a, b1 , b2 , and b3) were significant for all characters except the number of branches per plant and the dry weight of vegetative growth, the value $\mathrm{c}$ was significant for all characters except the number of branches per plant , while the value of $d$ was significant for all characters except the number of branches per plant and the total chlorophyll in leaves, the $\sqrt{ } \mathrm{H} 1 / \mathrm{D}$ was less than one for the number of leaves per plant, leave area and leave area index , the value of $\left(\mathrm{p}^{-} \mathrm{q}^{-}\right)$was less than 0.25 for all traits , the value of $\mathrm{KD} / \mathrm{KR}$ was more than one for all characters except the dry weight of vegetative growth. The broad sense heritability was higher for all characters, the line regression between $(\mathrm{Wr} / \mathrm{Vr})$ cut the head line over the original point for all characters.
\end{abstract}

Key words: diallel analysis, genetic architecture, Hayman method, peas.

دراسة البنية الوراثية في البسله باستخدام تحليل Diallel بطريقة Hayman (ا) للنمو الخضري

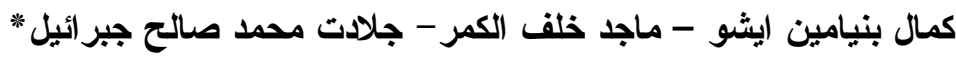

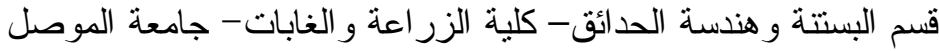

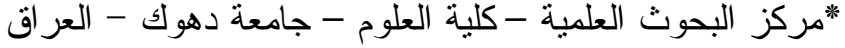

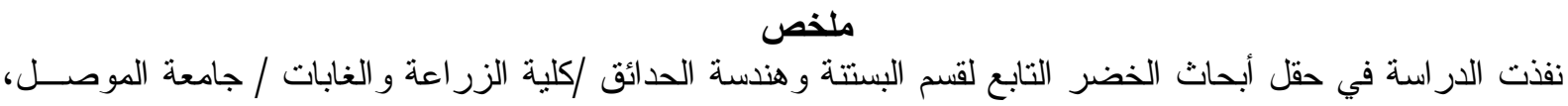

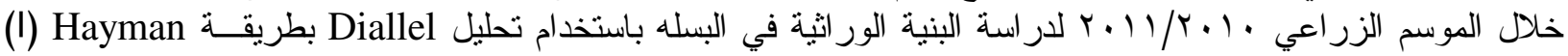

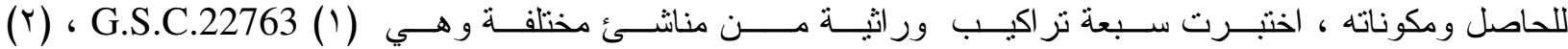

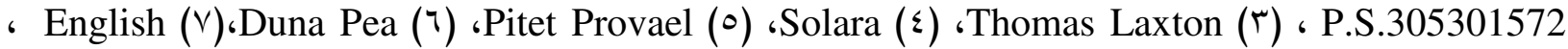

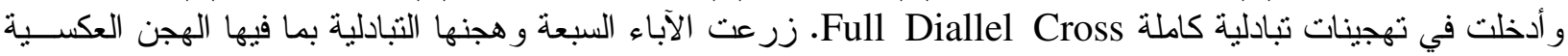

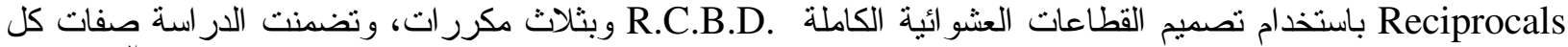

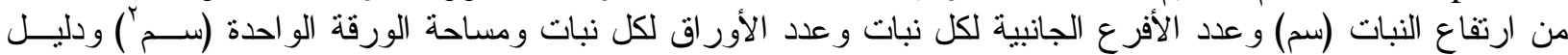

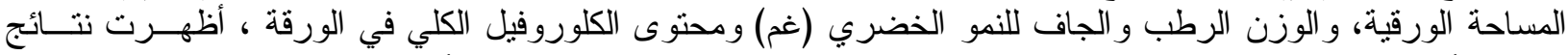

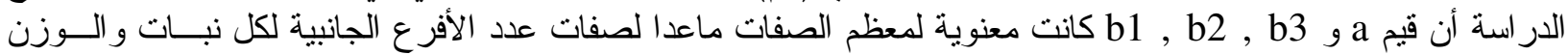


الجاف للنمو الخضري ، وكانت قيم c معنوية لمعظم الصفات المدروسة ماعدا لصفة عدد الأفرع الجانبية لكل نبات وان قيم الكيم

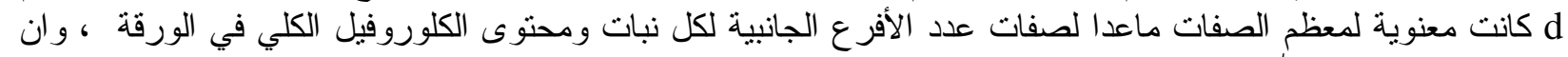

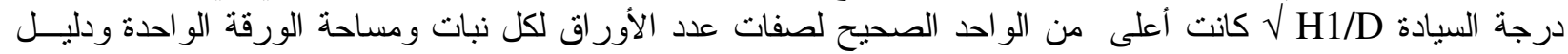
المساحة الورقية ، وان قيم ((

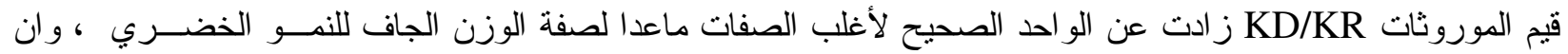

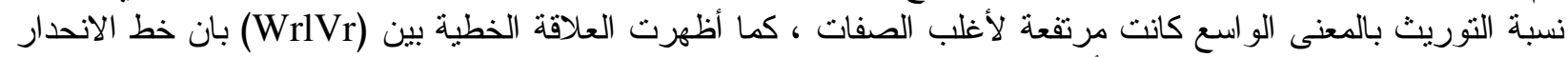

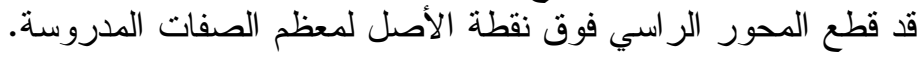

آخرون (q Lila

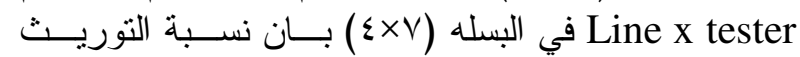

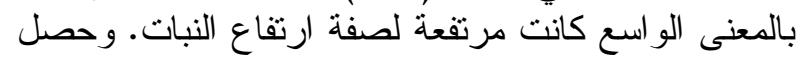

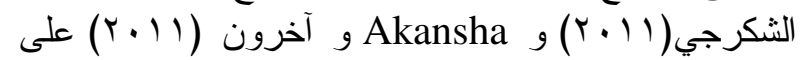
نسبة التوريث بالمعنى الو اسع مرتفعة لصفة الصنا ارتفاع النبات

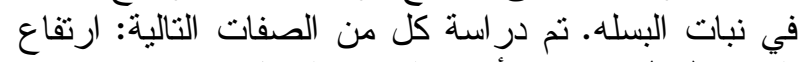

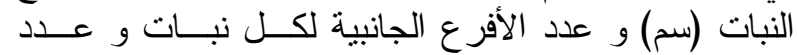

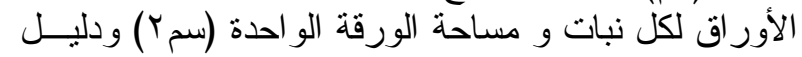

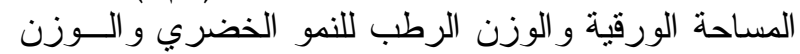

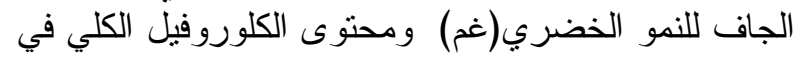
الورقة.

يهدف هذا البحث إلى دراسة البنية الور اثية في نبــات

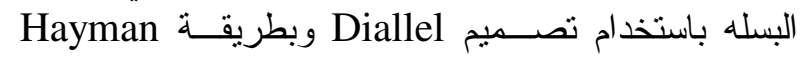
(1954) للنمو الخضري.

\section{r r مواد وطر ائق البحث}

واستخدم في الدر اسة سبعة نر اكيب ور اثنية تمثل سلالات

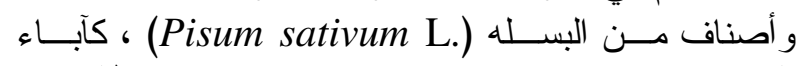

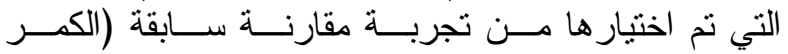

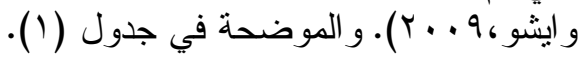

الجدول (1): أسماء ومصادر الأصناف والسلالات المستخدمة

\begin{tabular}{|c|c|c|}
\hline \multicolumn{3}{|c|}{ في الدراسة. } \\
\hline المصدر & الصنف أو السلالة & التجرية في \\
\hline ICARDA (مركز بحوث نينوى) ICA & G.S.C.22763 & I \\
\hline ICARDA (مركز بحوث نينوى) ICA & P.S.305301572 & $\mathrm{r}$ \\
\hline الأسواق المحلية & $\begin{array}{l}\text { Thomas } \\
\text { Laxton }\end{array}$ & $\Gamma$ \\
\hline ICARDA (مركز بحوث نينوى) ICA & Solara & $\xi$ \\
\hline محافظة دهوك & Petit Provael & o \\
\hline ASTURALIA & Duna Pea & 7 \\
\hline الأسواق المحلية & English & $\mathrm{v}$ \\
\hline
\end{tabular}

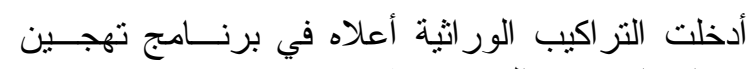

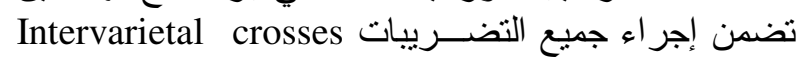

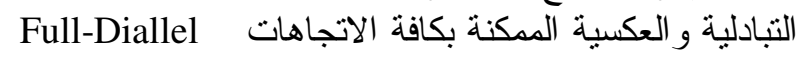
crosses وفق الطريقة الأولى Model I والموديل الأول

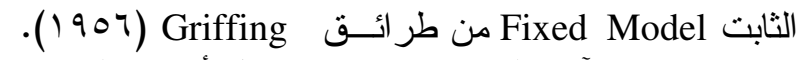
زرعت بذور الآباء السبعة مباشرة في حقل أبحاث الخضر الزبر

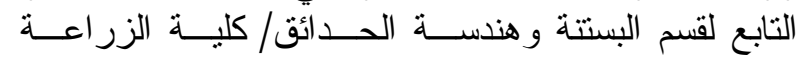

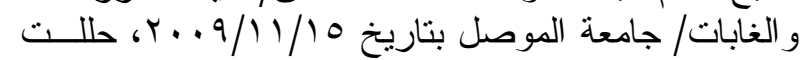

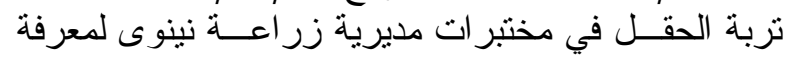

\section{1 المقدمة}

تعد البسله (Pisum sativum L.) peas أحد نباتـات

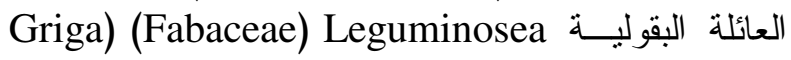

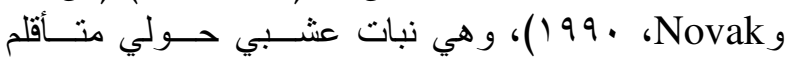

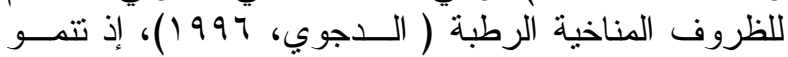

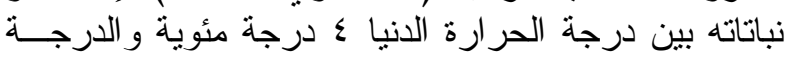

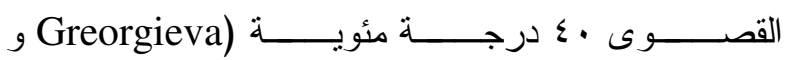
Diallel Lichtenthaler فكرة لاختبار قاعدة ور اثية جيدة للآباء (Murray) و آخرون

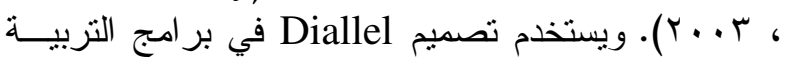

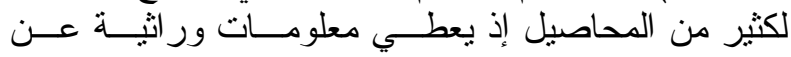

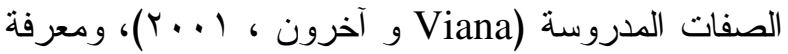

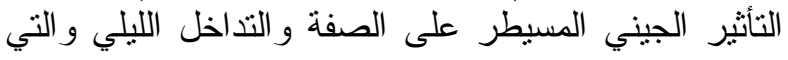

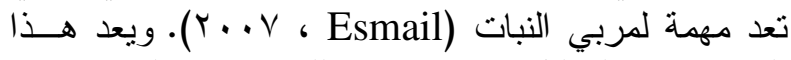

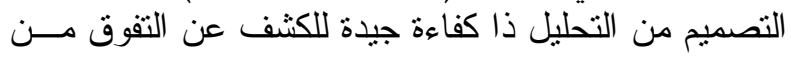

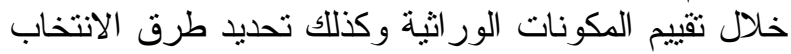

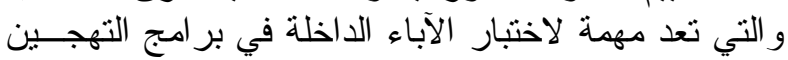

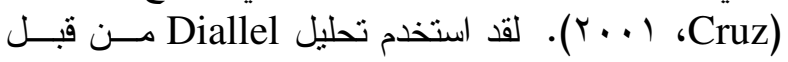

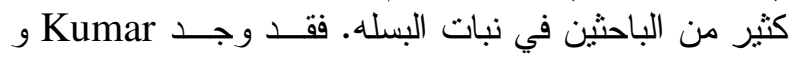

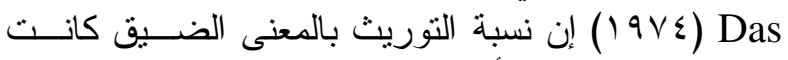

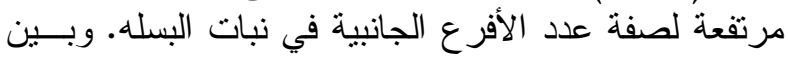
Kumar

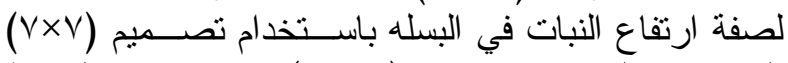

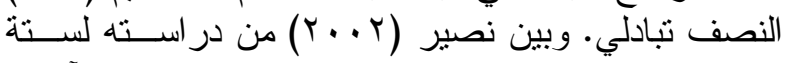

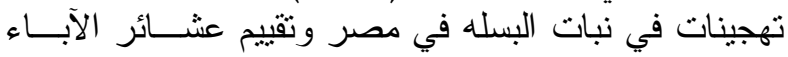

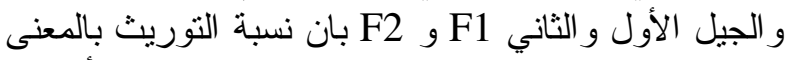

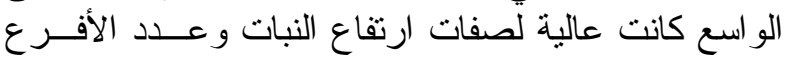

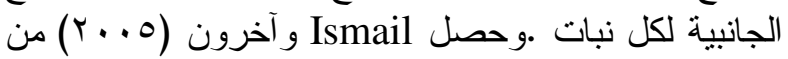

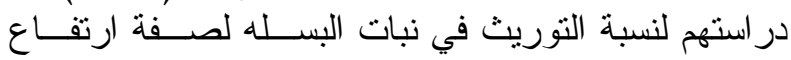

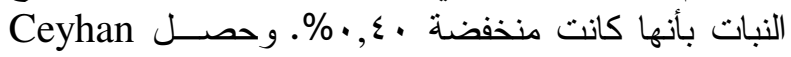

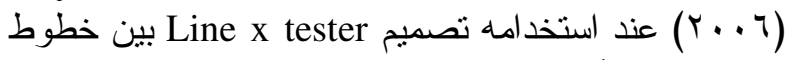

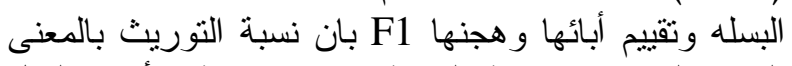

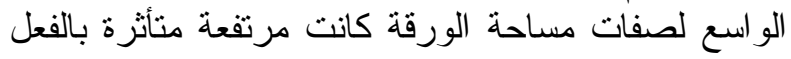

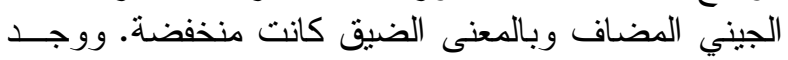
Chadha

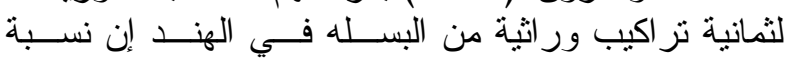

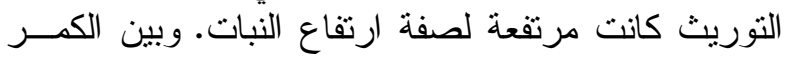

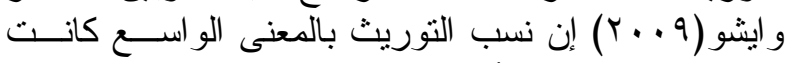

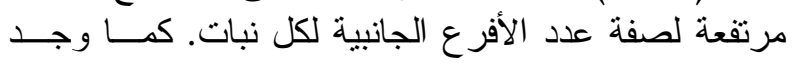




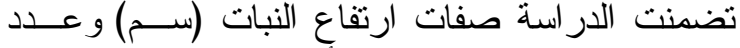

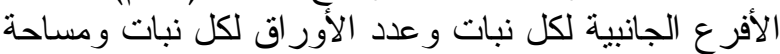

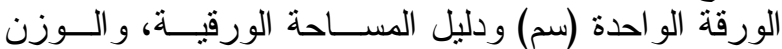

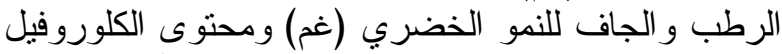

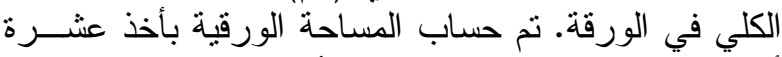

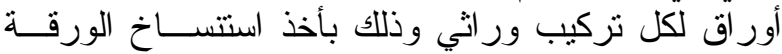

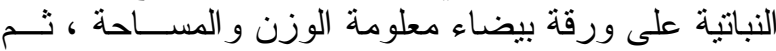
حساب مساحة الورقة من القانون التالي:

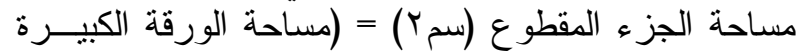
× وزن الجزء المقطوع)/وزن الورقة الكوعة الكبيرة (المعلومة)، .(199. ، S Saieed) تم تقدير محتوى ، الكلوروفيل الكلي باســتخدام الجهــاز

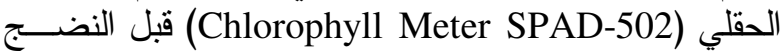

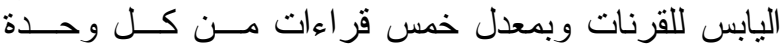
تجريبية. كما قدرت نسب التوريث بالمعنيين الو اسع (b.s)

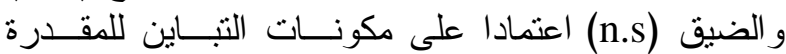

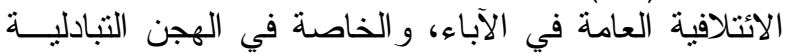

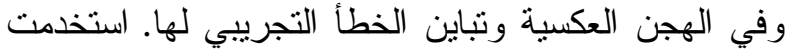
طريقة تحليل الهجن التبادلية المقترحة من قبل

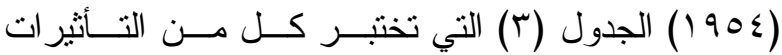

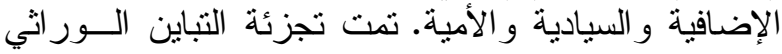

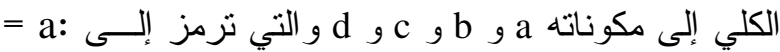

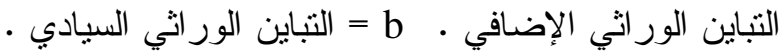

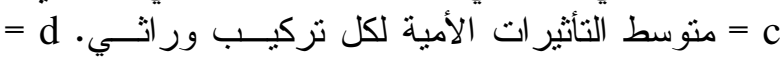

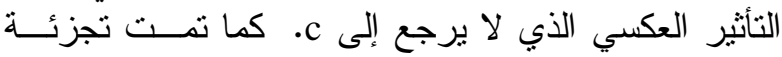

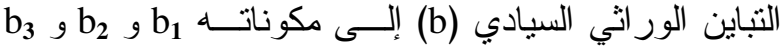

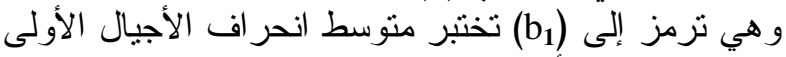

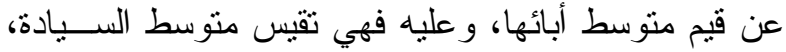

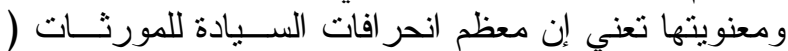

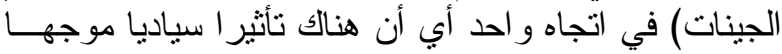
Directional Dominance Effect الانحر افات السيادية للأجيال الأولى عن قيم متوسط الآباء

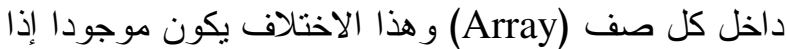

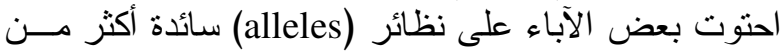

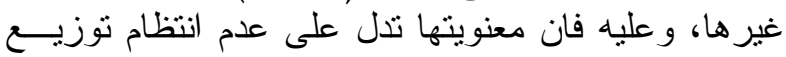

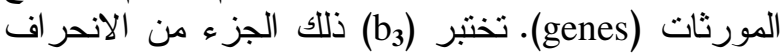

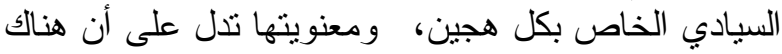

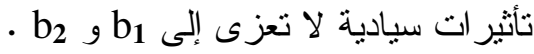

الخصائص الكيميائية و الفزيائية لها كما في الجدول (ץ) •

جدول (r): الخصائص الكيميائية والفيزيائية لتربة الحقل للموسم

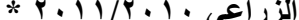

\begin{tabular}{|c|c|c|c|}
\hline \multicolumn{2}{|c|}{ الخصائص الفيزيائية } & \multicolumn{2}{|c|}{ الخصائص الكيميائية } \\
\hline$\varepsilon \neg, \vee 0$ & الرمل \% & 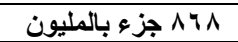 & النتروجين \\
\hline$r q, \leqslant r$ & السلت & ا I , Y جزء بالمليون & الفسفور \\
\hline $17, \wedge r$ & الطين & ه 1, 1 جزء بالمليون & البوتاسيوم \\
\hline \multicolumn{2}{|c|}{ نسجة التربة } & $\cdot, r$ & $(1: 1) \mathrm{Ec}$ \\
\hline \multirow{2}{*}{\multicolumn{2}{|c|}{ لومية }} & $v, \varepsilon$ & $(1: 1) \mathrm{pH}$ \\
\hline & & $1,9 v$ & $\%$ O.M \\
\hline
\end{tabular}

*ققسم المختبرات والدراسات التطبيقية ، مديرية زراعة نينوى ، وزارة الزراعة

زرعت r-ـ بذور في الجورة الواحدة وكانت المسافة

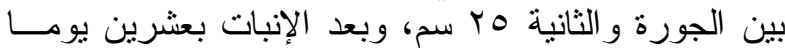

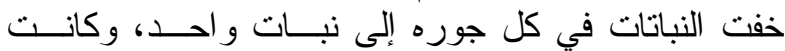

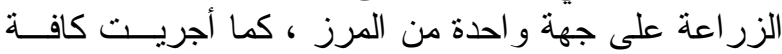

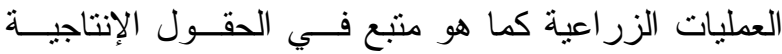

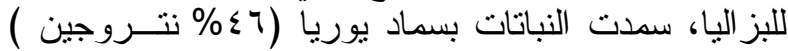

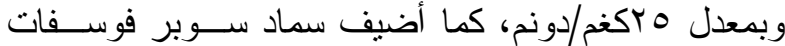

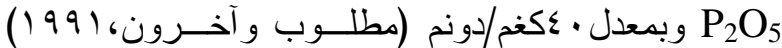

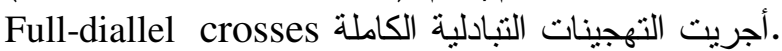

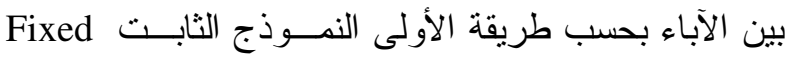
( Griffing) Model I

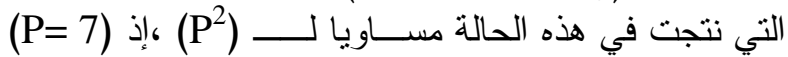

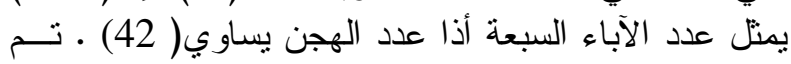

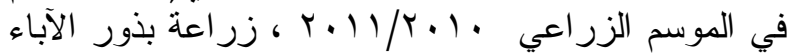

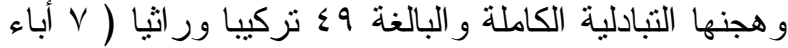

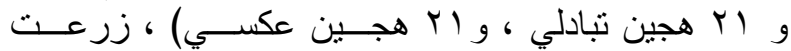

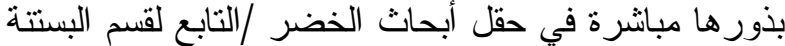

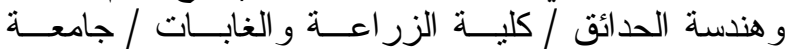

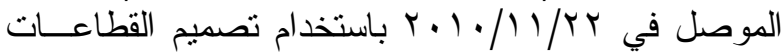

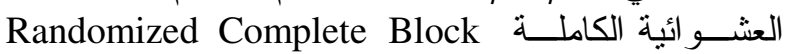
وبثلاث مكررات (الر اوي وخلــ Design (R.C.B.D.)

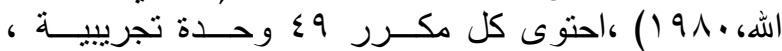

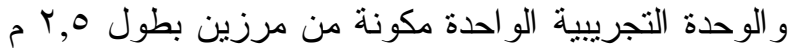

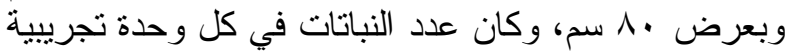

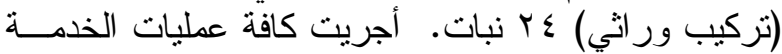

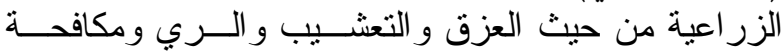

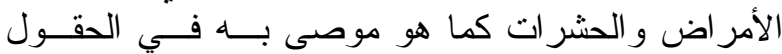
الإنتاجية لنبات البسله (مطلوب و آخرون ، 919 (1).

الجدول (rّ): طريقة تحليل التباين حسب Hayman (ع ه9 1 ).

\begin{tabular}{|c|c|c|c|}
\hline S.O.V & d.f & s.s & Constants \\
\hline $\mathbf{A}$ & n-1 & {$\left[\sum(\mathbf{y r} .+\mathbf{y} . \mathbf{r})^{2} / 2 \mathrm{n}\right]-\left(2 \mathbf{y} . .^{2}\right) / \mathbf{n}^{2}$} & $\int \mathbf{T i}$ \\
\hline b & $1 / 2 \mathbf{n}(\mathbf{n}-1)$ & {$\left[\sum\left(\mathrm{y}_{\mathrm{rs}}+\mathrm{y}_{\mathrm{sr}}\right)^{2} / 2\right]-\left[\sum(\mathrm{yr} .+\mathrm{y} . \mathrm{r})^{2} / 2 \mathrm{n}\right]+\left(\mathrm{y} . .^{2}\right) / \mathrm{n}^{2}$} & JJrs \\
\hline $\mathbf{b}_{1}$ & 1 & $(\text { y.. }-\mathbf{n y} .)^{2} / \mathbf{n}^{2}(\mathbf{n}-1)$ & \\
\hline $\mathbf{b}_{2}$ & n-1 & {$\left[\sum(\mathbf{y r} .+\mathbf{y} \cdot \mathbf{r}-\mathbf{n} \mathbf{y r})^{2} / \mathbf{n}(\mathbf{n}-2)\right]-\left[(2 \mathrm{y} . .+\mathrm{n} \mathbf{y} .)^{2} / \mathbf{n}^{2}(\mathbf{n}-2)\right]$} & $\mathbf{R}$ \\
\hline $\mathbf{b}_{3}$ & $1 / 2 \mathbf{n}(\mathbf{n}-3)$ & $\mathbf{b}-\mathbf{b}_{1}-\mathbf{b}_{2}$ & Rs \\
\hline c & n-1 & {$\left[\sum(\mathbf{y r} .+\mathbf{y} . \mathbf{r})^{2} / 2 \mathrm{n}\right]$} & $\mathbf{K r}$ \\
\hline $\mathbf{d}$ & $1 / 2(n-1)(n-2)$ & {$\left[\sum\left(\mathbf{y}_{\mathrm{rs}}+\mathrm{y}_{\mathrm{sr}}\right)^{2} / 2\right]-\left[\sum(\mathrm{yr} .+\mathbf{y} \cdot \mathbf{r})^{2} / 2 \mathrm{n}\right]$} & Krs \\
\hline Total & $n^{2}-1$ & $\left.\sum \mathrm{yrs}^{2}-\left(\mathrm{y} . .^{2}\right) / \mathbf{n}^{2}\right]$ & \\
\hline
\end{tabular}


ولمجموع المكررات ، ونم اختبار ها ضد التداخل بــين أي (الي

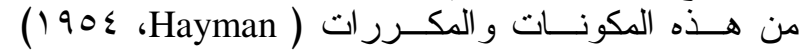

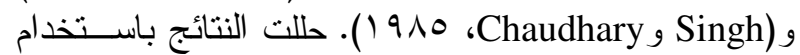

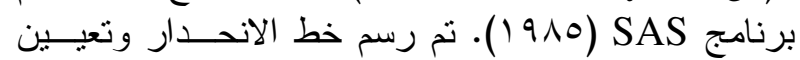

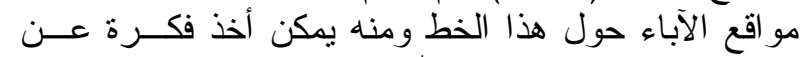

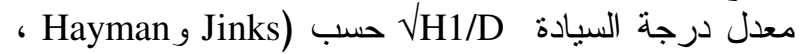

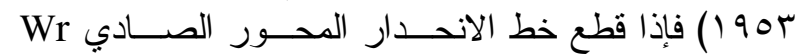

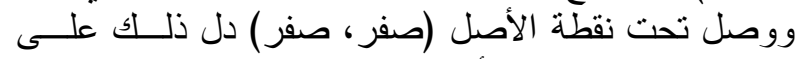

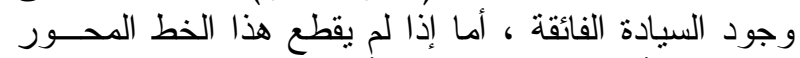

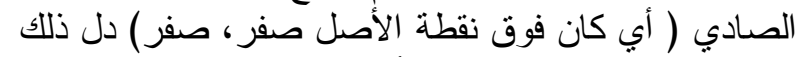

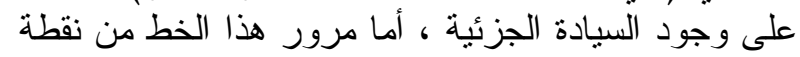

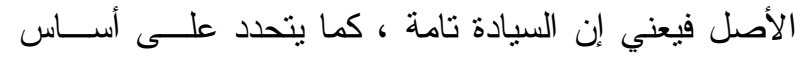

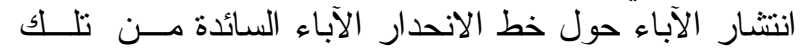

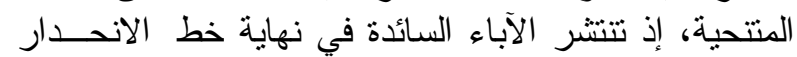

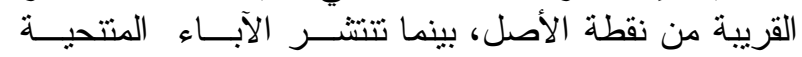

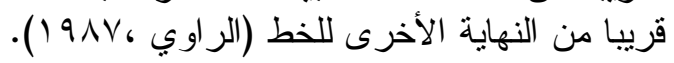

\section{r. أنتائج و المناقثة}

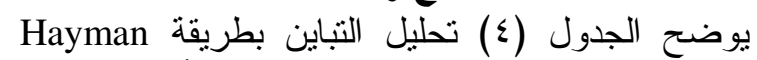
(90 19 ) لصفات ارتفاع النبات (سم) و عدد الأفرع ع الجانبية

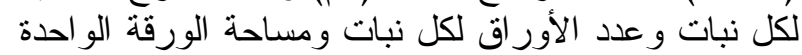

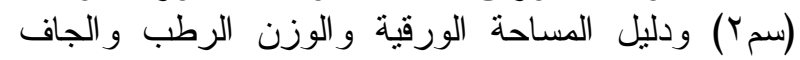

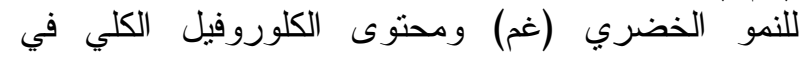
الورقة. يلاحظ فيه إن قيم (a) التي تقيس متوسط التئن التباين

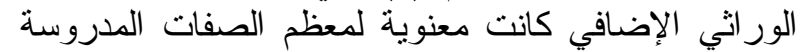

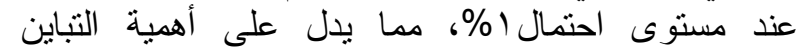

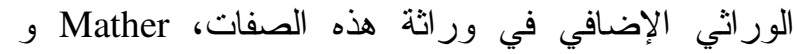
إلى Jinks

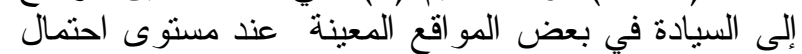

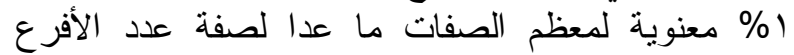

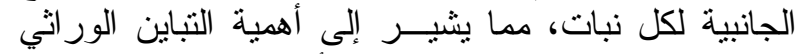

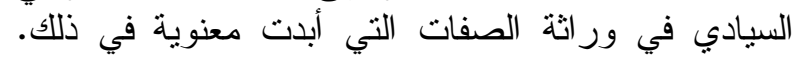

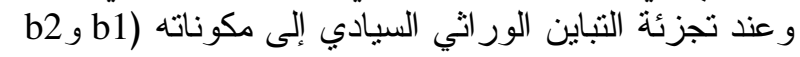

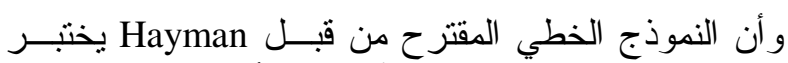

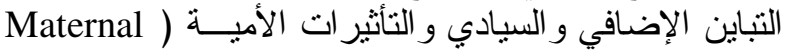
(effects حيث أن:

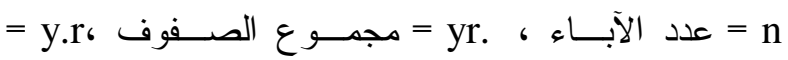

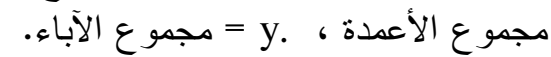

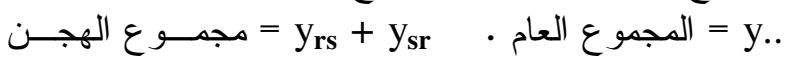

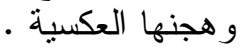

= ysr - yrs

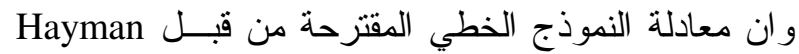

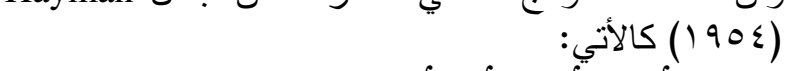
$\mathrm{yrs}=\mathrm{M}+\mathrm{Jr}+\mathrm{Js}+\mathrm{Jrs}+\int+\int \mathrm{r}+\int \mathrm{s}+\int \mathrm{rs}+2$ $\mathrm{Kr}+2 \mathrm{Ks}+2 \mathrm{~K}$ rs

حيث أن :

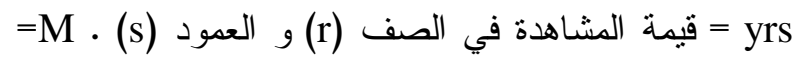
المتوسط العام (منوسط المجتمع). ال متوسط الانحر اف عن المتوسط العام و العائد لصفوف ل Jr الآباء.

Js الآباء.

Jrs العكسية.

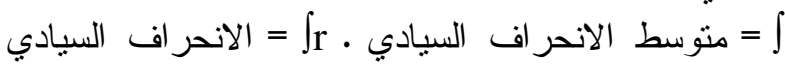
الأخر العائد لصفوف (r) الآباء.

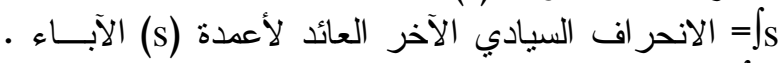
= Jrs كاب =2Kr كأب ذكري و أب أنثوي. 2ks = الاختلافات بين" تأثير ات أعمدة الآبــاء المسـتـعلة كأب ذكري و كأب أنثوي. 2krs

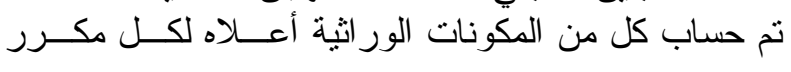

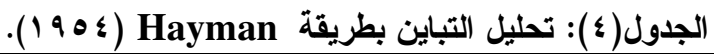

\begin{tabular}{|c|c|c|c|c|c|c|c|c|c|}
\hline الكلي في الورقة & اللنموزن الخضري (غر) & اللنمون الخضري الرطب & الليل المساحة & مساحة الورقة & / عدات الأوراق & | عدانب الأفرعة & 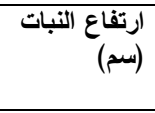 & $\begin{array}{r}\text { الدرية } \\
\text { d.f } \\
\text { d.f }\end{array}$ & $\begin{array}{r}\text { التباين } \\
\text { S.O.V }\end{array}$ \\
\hline$* * 017,701$ & 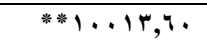 & $* * \Gamma q \triangle \curlyvee q, \Gamma$ & $* * \| T, \cap \wedge r$ & $* * V \leqslant 0,4 \wedge 1$ & 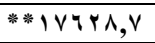 & $* * r, q \vee \leq$ & ***VAYr,Y & 7 & $\mathbf{a}$ \\
\hline * $1 \leqslant, \ldots \varepsilon$ & $* * \bullet V \cdot, q 9$ & 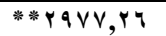 & $* 0, \xi \wedge \Gamma$ & "IY & $* * \varepsilon \leqslant \leq \leqslant, 79$ &., 095 & 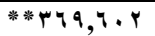 & rI & B \\
\hline$* * \sqcap ৭, \Gamma \leq 0$ & rir,q०q & $* * q \curlyvee \wedge \varepsilon, \cdot\{$ & $* * r 4, \cdot r$ & $* 170, r \cdot \Lambda$ & $* * r r V \leq q, \varepsilon$ & **\%, TrT & $* * r \vee q \vee, \wedge 1$ & 1 & $b_{1}$ \\
\hline$q, \wedge r r$ & $1+., 90$ & ***YIY & "* & $7 \leqslant, \leqslant 79$ & 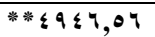 & $\cdot, \xi \vee \vee$ & * $1 \leqslant 0,7 Y_{0}$ & 7 & $\mathbf{b}_{2}$ \\
\hline$* 1 \leqslant, 797$ & $* * \vee \wedge \wedge, \cdot \wedge 1$ & $* * \because \wedge \wedge \cdot, \vee \vee \uparrow$ & *r,, $\mathrm{Q} \odot \mathrm{r}$ & $* 101, .1 \%$ & 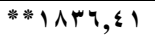 &., $01 \mathrm{~V}$ & $* * Y Y \cdot, V Y$ & $1 \varepsilon$ & $\mathbf{b}_{3}$ \\
\hline$* * 0, \mu \mid V$ & $* * 1 \Gamma \mid \vee, 10$ & $* * Y \leqslant \leqslant \varsigma, \wedge \vee$ & $* v, 109$ & ***YYY,TAI & ***1, RY, I & $\cdot$, YYY & $* 1 \wedge \leqslant, \cdot \leq Y$ & 7 & C \\
\hline $1,, \leqslant \vee 4$ & ***rq,人। & $* * r .79,0 r$ & $* v, \cdot r \wedge$ & ***19.,Y & ***YYV,Vq &., 0.9 & **r.r,qr। & 10 & D \\
\hline$\varepsilon, \wedge \leftrightarrow \checkmark$ & $7 V, 017$ & $117, V H Y$ & $\cdot, \cdot V r$ & $0, .9 V$ & $V \cdot, 9 Y \leqslant$ & . YRY & $10,1 \leqslant 4$ & Ir & $\mathbf{B a}$ \\
\hline $0, \leqslant \vee q$ & $\Delta ৭, r \leqslant r$ & $r \cdot r, \cdot r V$ & $\cdot, 19 \mathrm{~V}$ & 0,741 & $7 \cdot, 1 \cdot 1$ & ., YА५ & $r Y, \cdot A r$ & $\leqslant Y$ & $\mathbf{B b}$ \\
\hline Ir,^q. & $\vee \wedge, \Gamma \Delta 1$ & $0 \leqslant, V \leqslant 1$ & •, & $\mid r, 991$ & $99,0 Y \xi$ & . & $0 ., \pi 19$ & $r$ & $\mathbf{B} \mathbf{b}_{1}$ \\
\hline$r, \wedge \leqslant q$ & $04,71 Y$ & $r \otimes \wedge, \cdot T r$ & $\cdot, r \leqslant r$ & $r, \leqslant \wedge \wedge$ & $r \varepsilon, 9 \vee r$ &., $1 \mu 1$ & $r \cdot, O r V$ & Ir & $\mathbf{B} \mathbf{b}_{2}$ \\
\hline $0,7 \leqslant 1$ & 09,100 & IA^,OHY & $\cdot, 141$ & $7, \cdot \wedge \wedge$ & $7 \Lambda, \cdot 70$ & . & ro,Vrr & $r \wedge$ & $\mathbf{B} \mathbf{b}_{3}$ \\
\hline$v, \leq 0 \leq$ & $90, r 9 \wedge$ & $r \leqslant 0,0 Y$ & $\cdot, 1 \leq$. & r,VYr & $\varepsilon V, \leqslant Y \leqslant$ & $\cdot, r V \varepsilon$ & $\mid \Lambda, r Y \Lambda$ & Ir & Bc \\
\hline$r, q \leq \varepsilon$ & $9 \varepsilon, \nabla \wedge 0$ & $1 \vee \wedge, 040$ & .,Yצ. & 0,711 & $r r, \wedge r q$ & $\cdot, \Upsilon \wedge \Delta$ & rI,. TV & $r$. & Bd \\
\hline
\end{tabular}



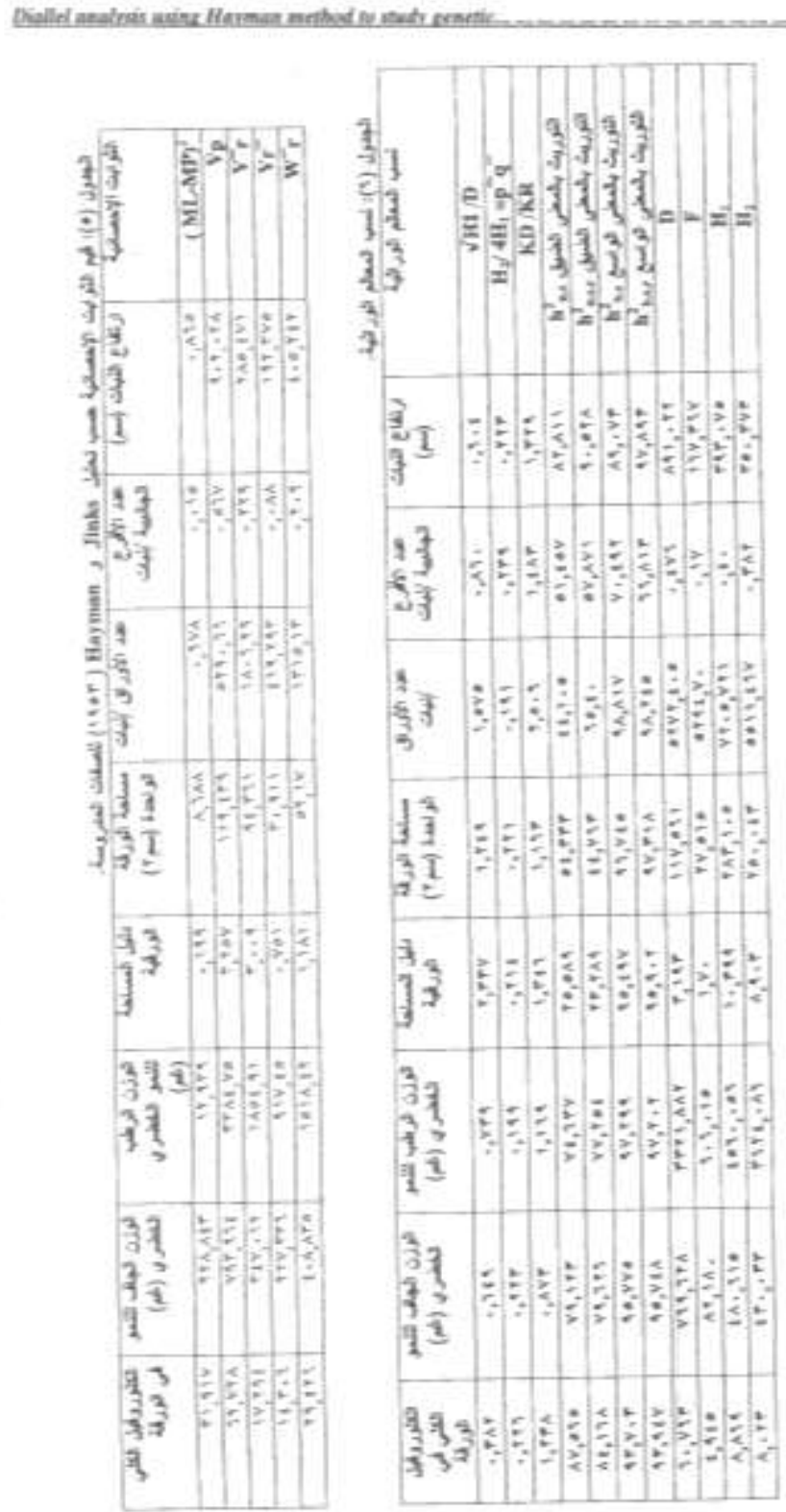

43 
الجاف للنمو الخضري إذ كانت سالبة. و اختلفت قيم التباين

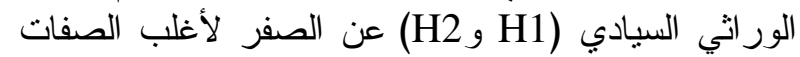

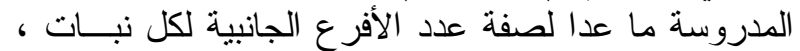

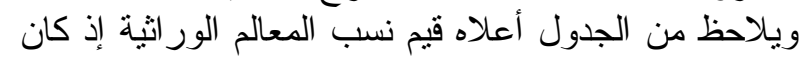

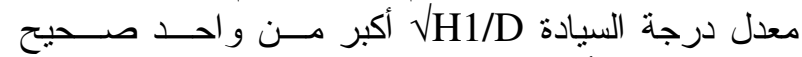

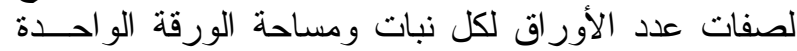

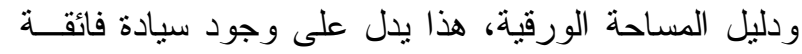
لهذه الصفات Over dominance

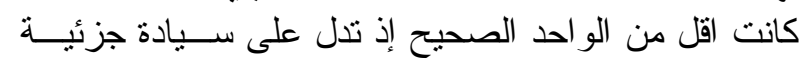

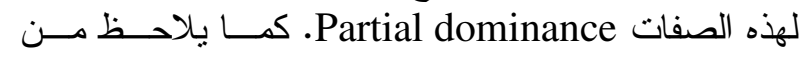

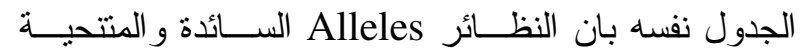

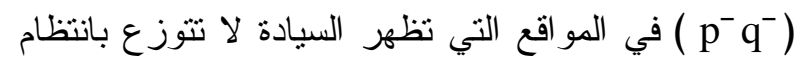

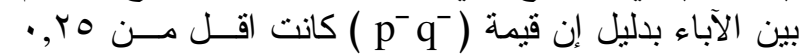

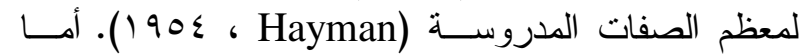

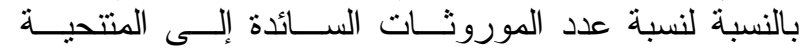
KD/KR

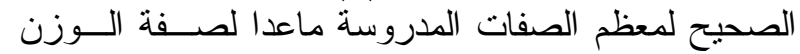

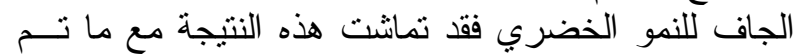

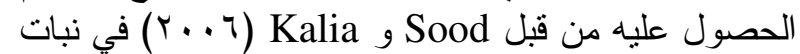

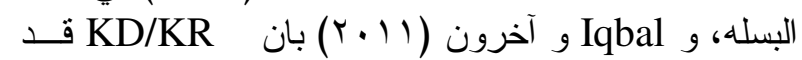

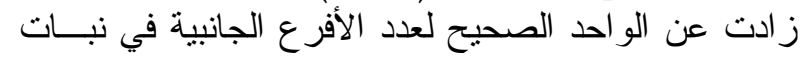

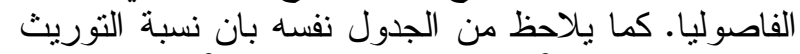

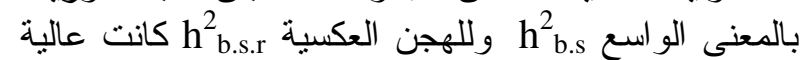

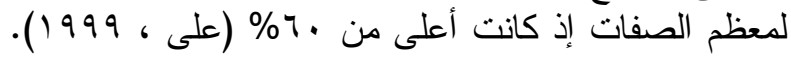

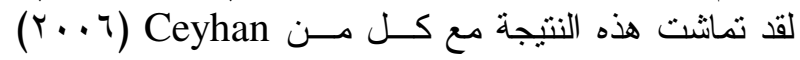

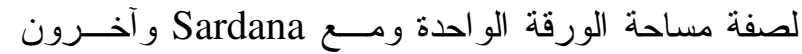
Chadha و و آخرون (Y...V)

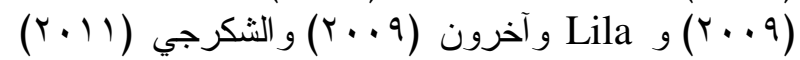

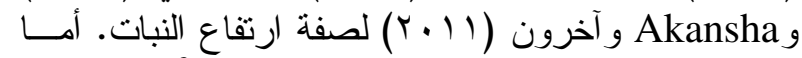

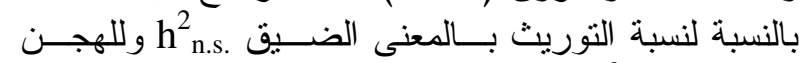

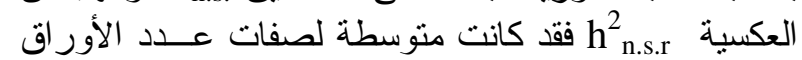
لكل نبات ودليل المساحة الورقية ومساحة الورقة الو الوتة الودة،

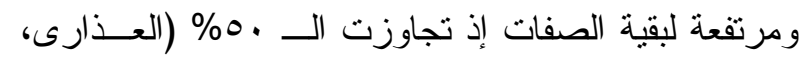

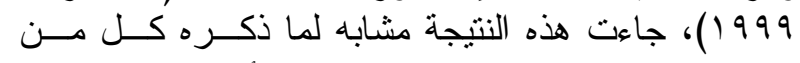
Kumar

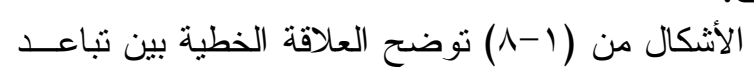

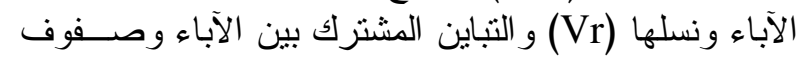

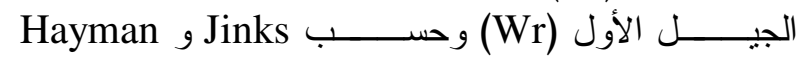

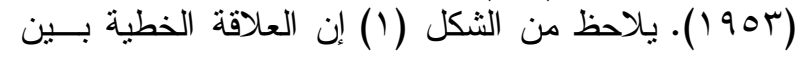

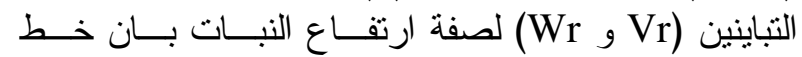
الانحدار قد قطع المحور الراسي فوق التون نقطة الأصل مشيرا

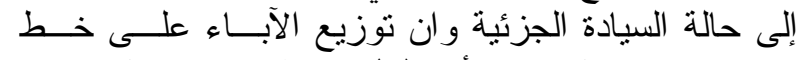

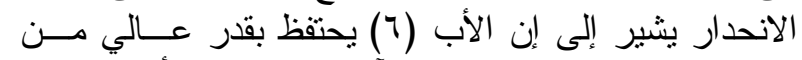

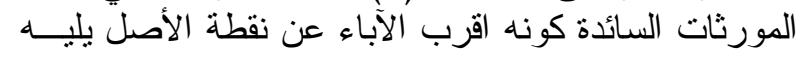

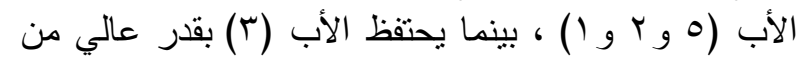
الموروثات المتتحية إذ يبدو ابعد الآباء عن نقطة الأبل الصل.

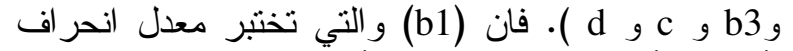

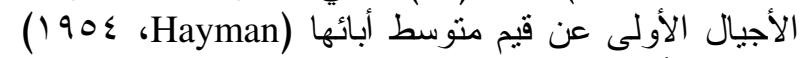

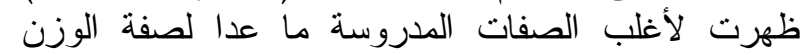

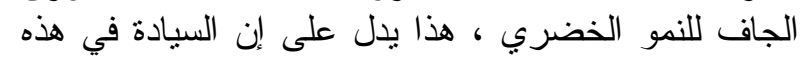

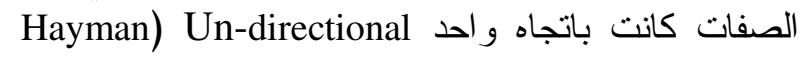

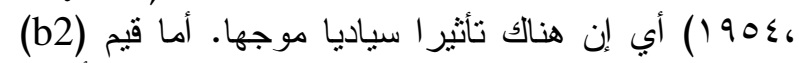

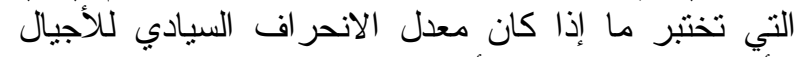

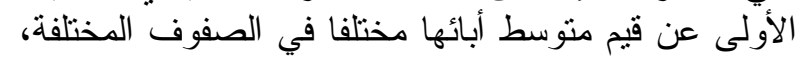

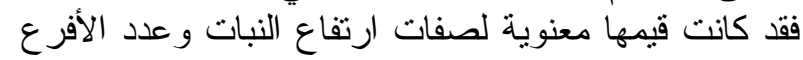

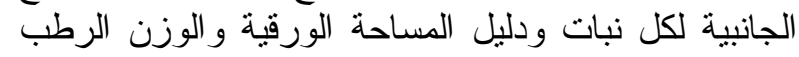

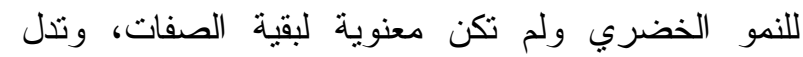

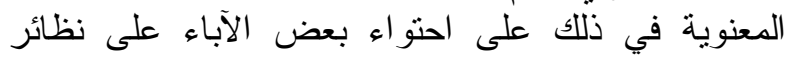

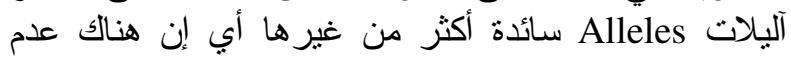
انتظام في توزيع الاليلات السائدة و المتتحية بين التين الآباء

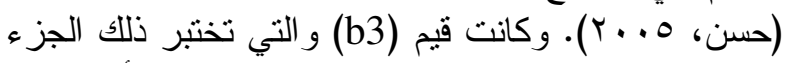

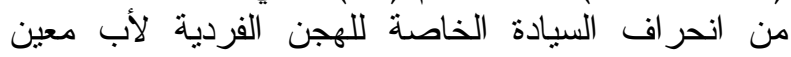
(F1's)

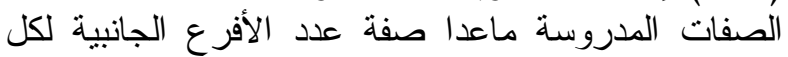

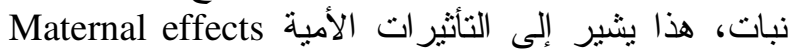

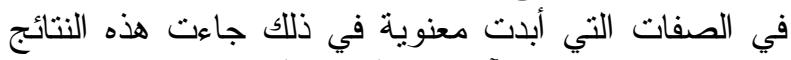

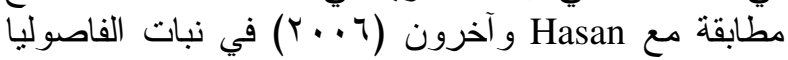

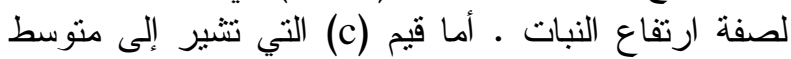

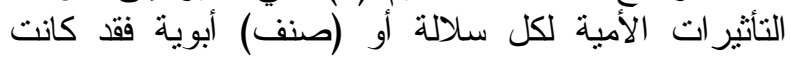

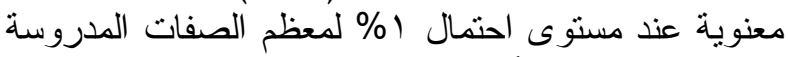

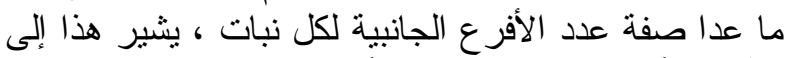

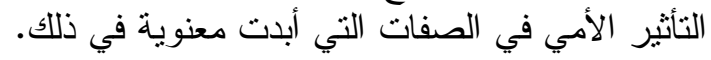

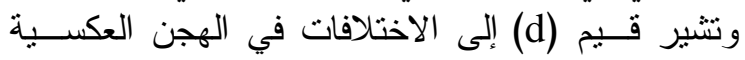

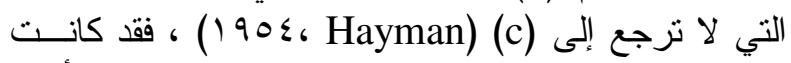

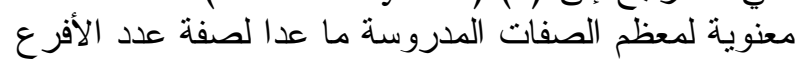

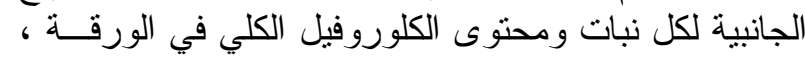

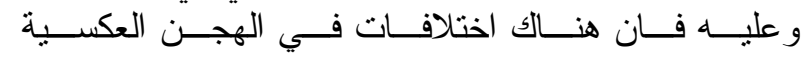
Reciprocals

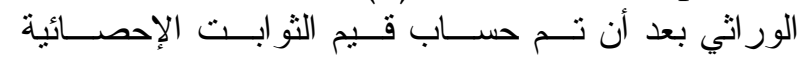

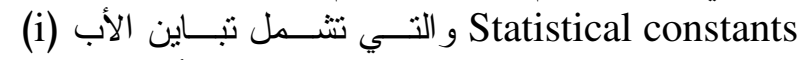

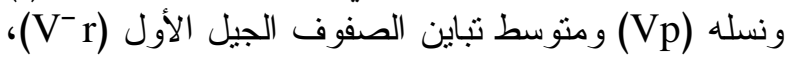

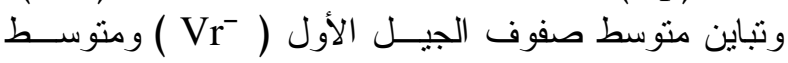

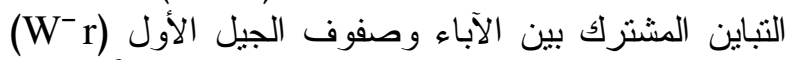

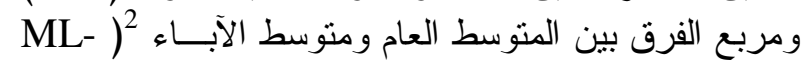
( ML0

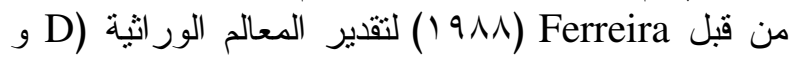

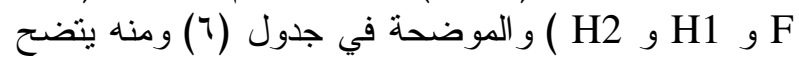

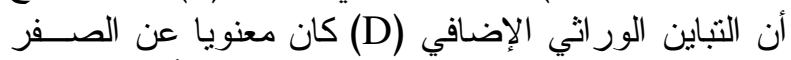

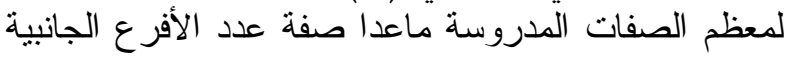

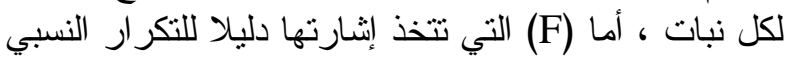

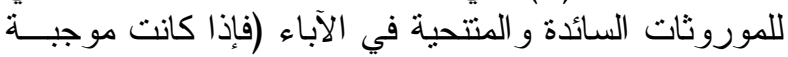

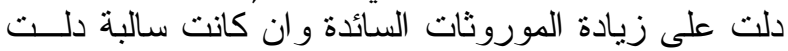

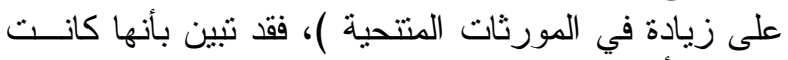

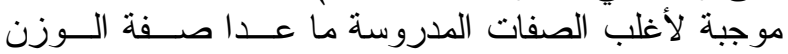



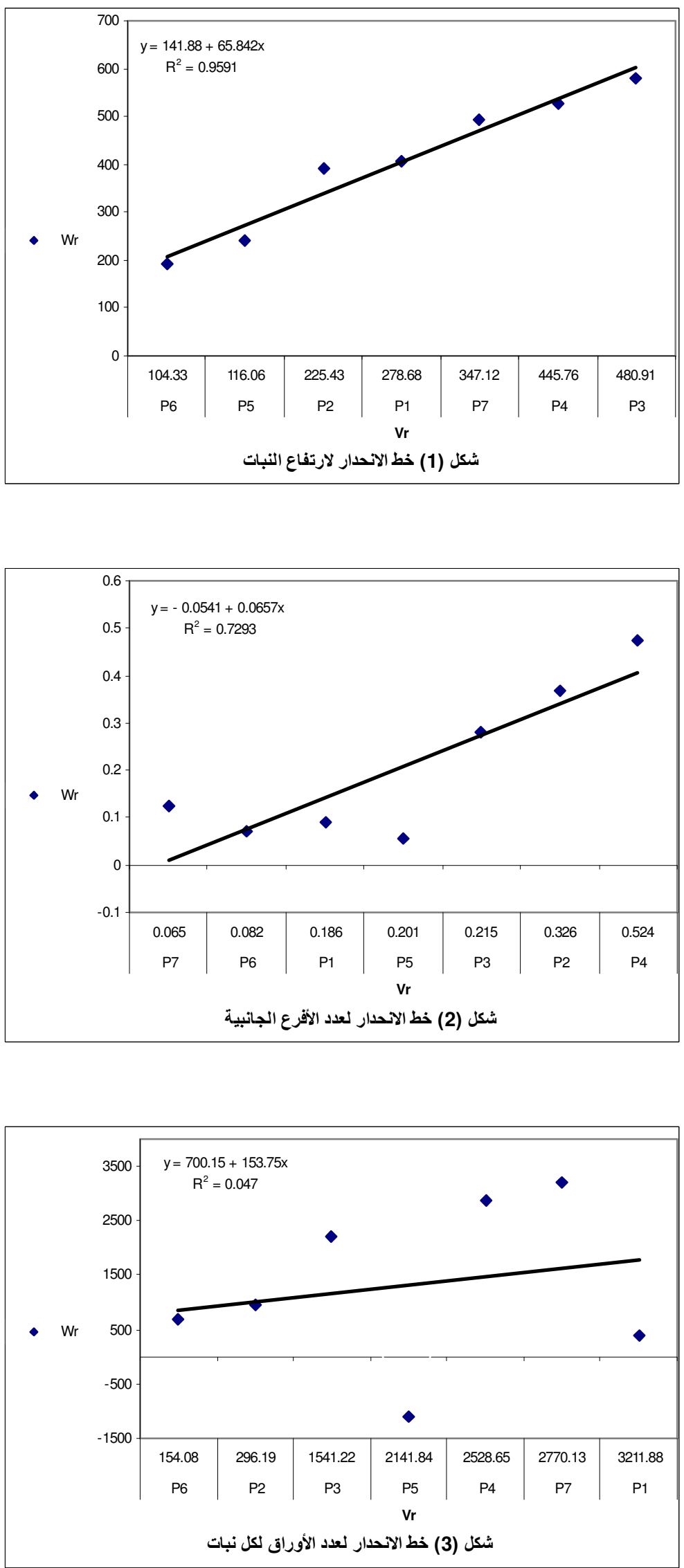


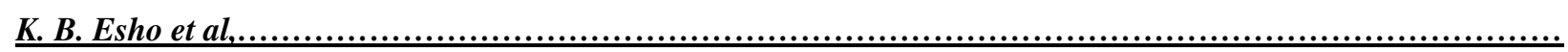
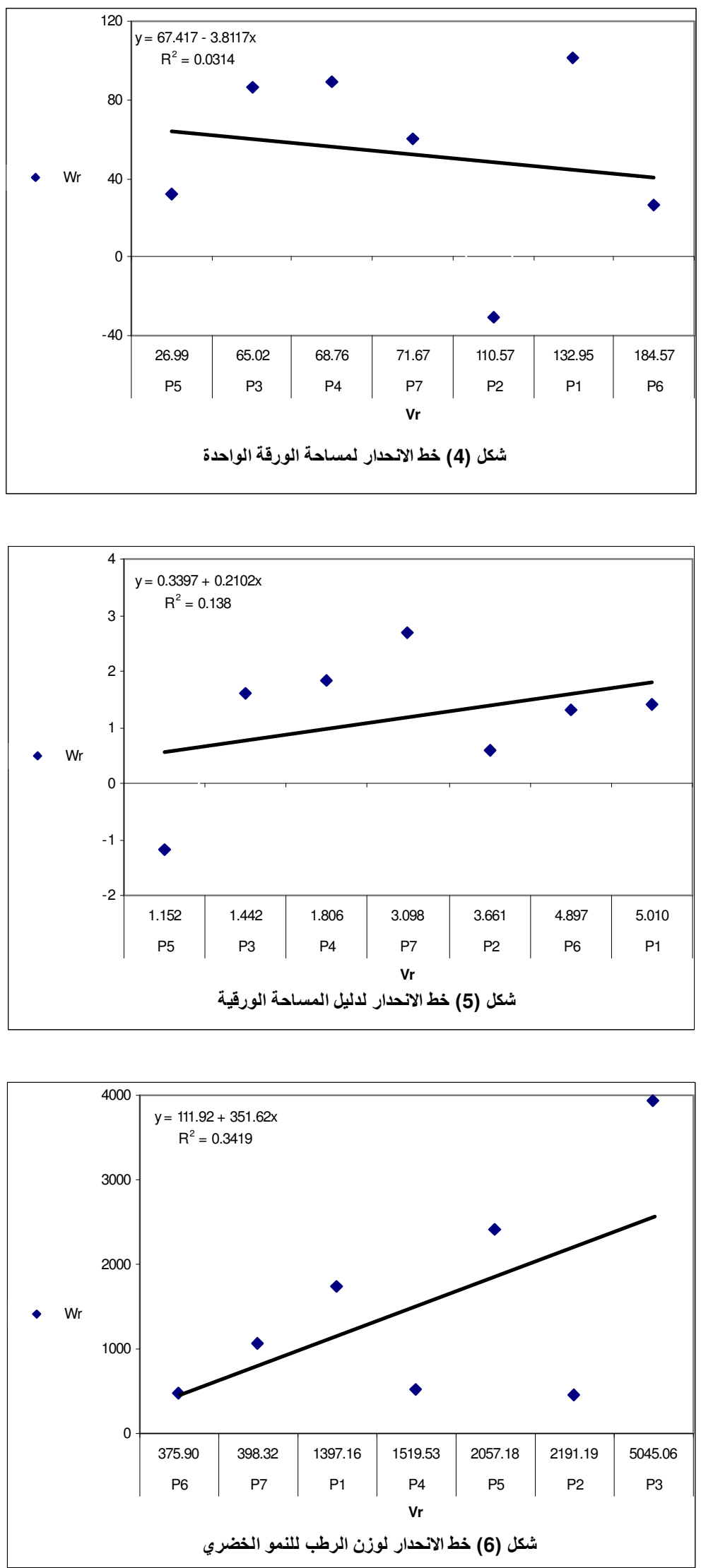

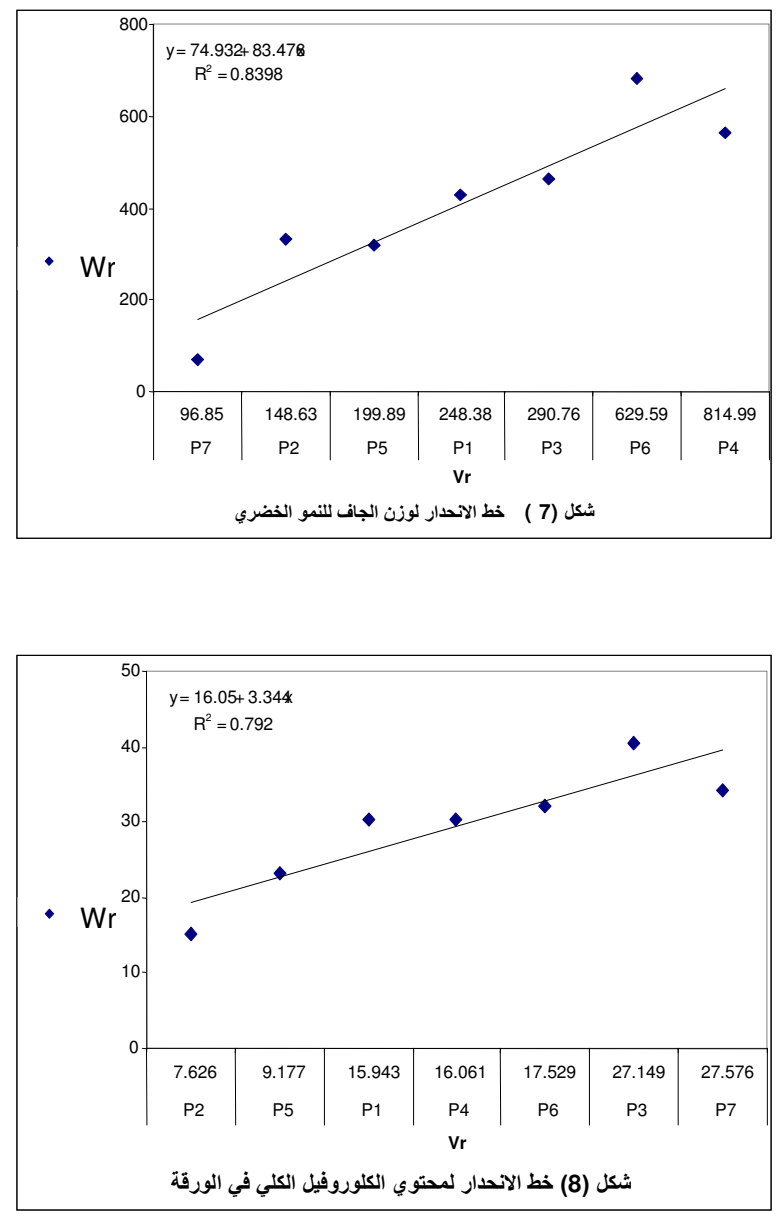

الآباء على خط الانحدار يبين إن الأب (Y) اقرب الآبــاء

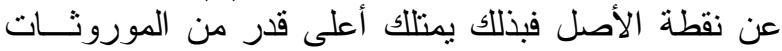

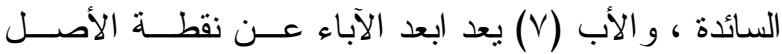

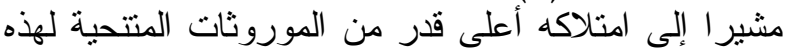

الصفة.

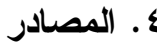

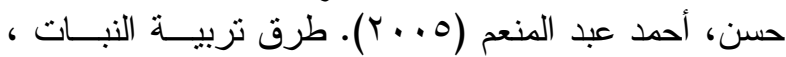

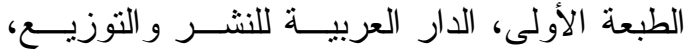

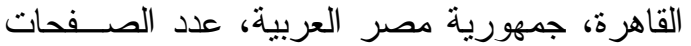

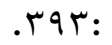

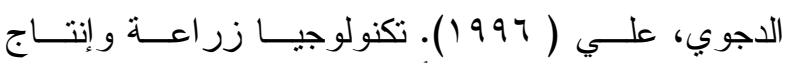

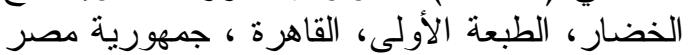

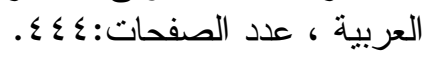

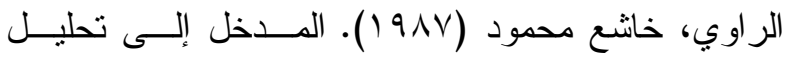

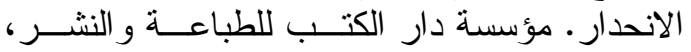

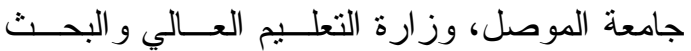

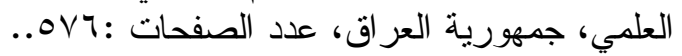

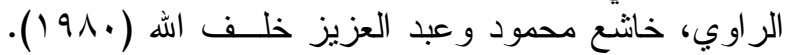

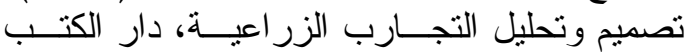

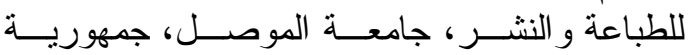

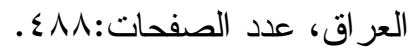

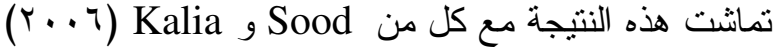

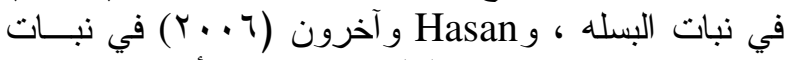

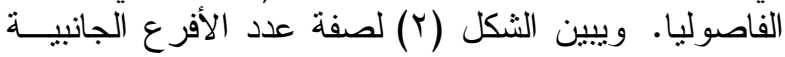

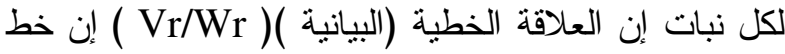

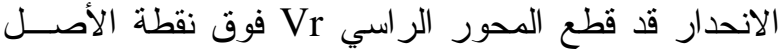

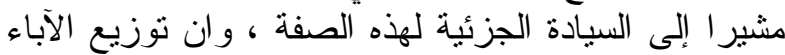

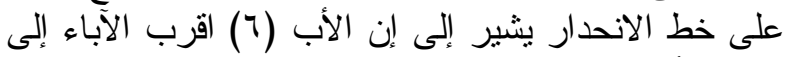

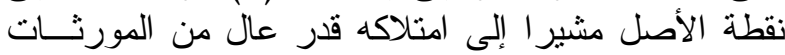

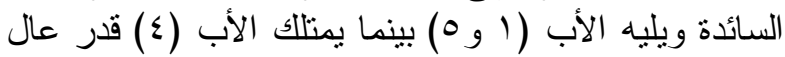
من المورثات المتتحية كونه ابعد الآباء عن نقطة الأبلاب الأصل.

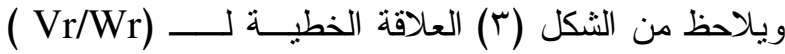

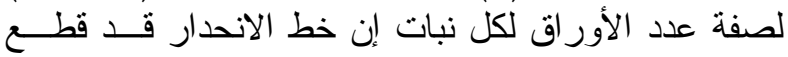

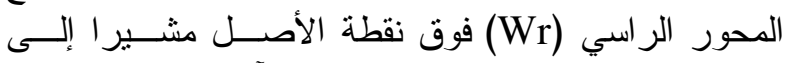

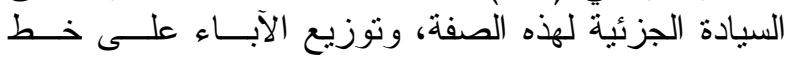

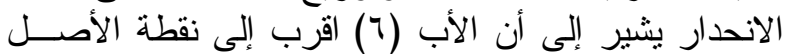

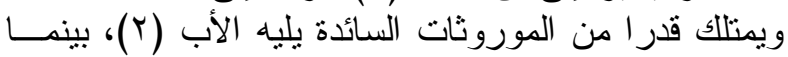

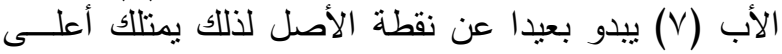

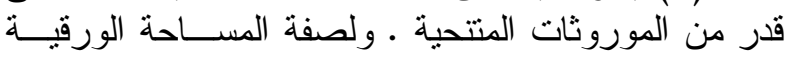

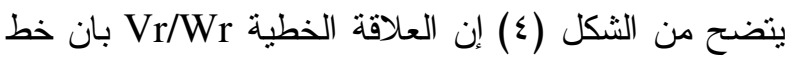
الانحدار قد قطع الدحور الر اسي فوق نقطة الأصل وتوزيع لإنيع

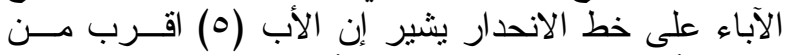

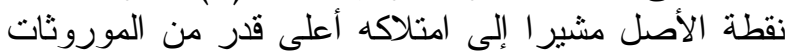

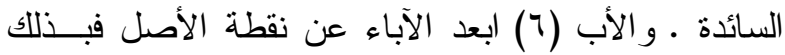

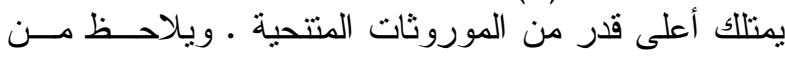

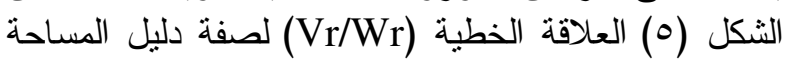

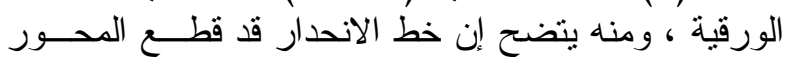

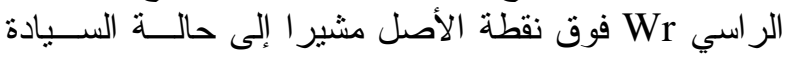

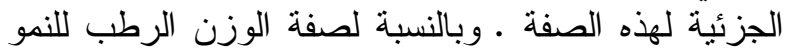

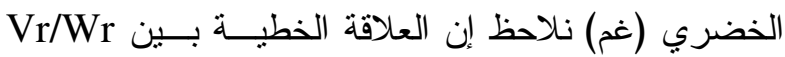

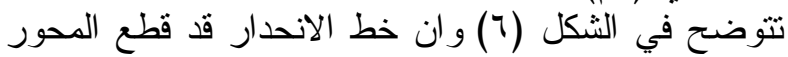

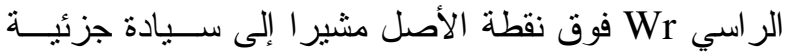

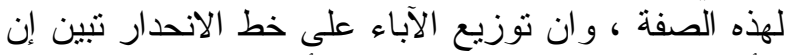

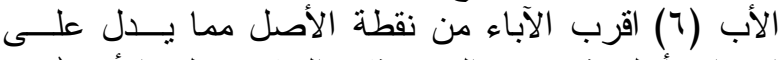

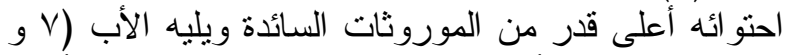

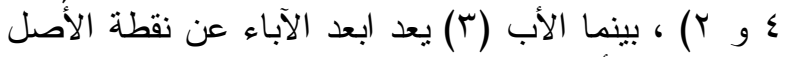

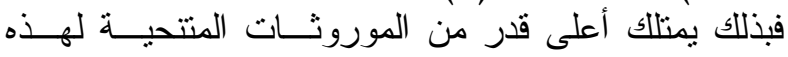

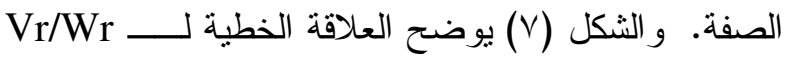
لصفة الوزن الجاف للنمو الخضري (غر) وفيه يتضح بان التحان

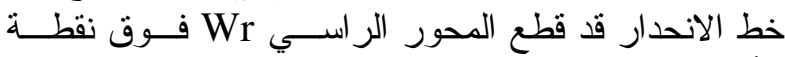

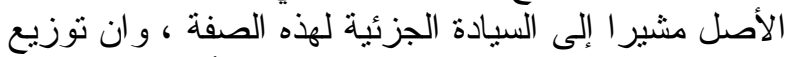

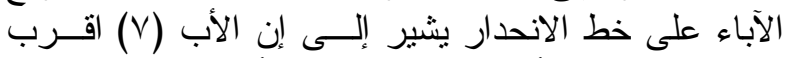

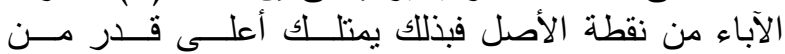

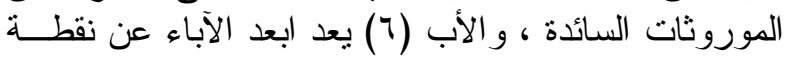

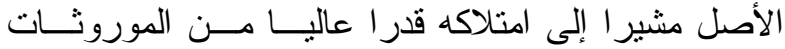

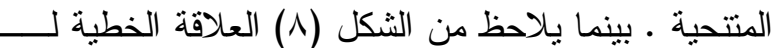

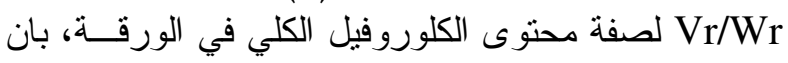

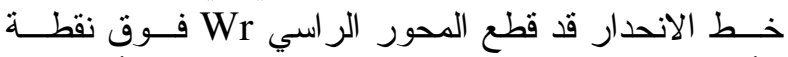

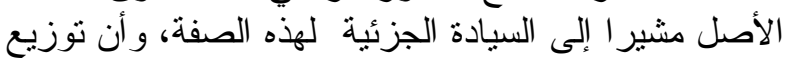


and its contributing traits in two interaspecific cotton crosses . J. Appl. Sci. Res., 3: 2075-2080 .

Ferreira P. E. (1988). A new look at Jink's Hayman method for estimation of genetical components in diallel crosses. Heredity, 6o: 347-353.

Greorgieva K. and Lichtenthaler H. K. (1999). Photosynthetic activity and acclimation ability of pea plants to low and high temperature treatment as studied by means of chlorophyll fluorescence. Journal of Plant Physiology, 155(3): 416-423.

Griffing B. C. (1956). Concept of general and specific combining ability in relation to diallel crossing systems. Australian of Biological Sci., 9:463-493.

Griga M. and Novak F. J. (1990) . Peas (Pisum sativum L.). In. Bajaj YDS (ed), Biotechnology in Agriculture and Forestry, 10, Legumes and Oilseed Crops P: 65-99.

Hayman B. I. (1954). The analysis of variance of diallel tables. Biometrics , $10: 235-244$.

Hasan M. N., Islam A. K. M. A., Mian M. A. K.and Hossain T. (2006). Inheritance of yield related traits in French bean (Phaseolus vulgaris L.). Gazipur, Bangladesh , 1-9 .

Iqbal A. M., Nehvi F. A., Wani S. A., Henna Q., Dar Z. A. and Lone A. A. (2011). Genetic studies in relation to yield and quality traits in rajmash (Phaseolus vulgaris L.). Journal of Plant Breeding and Crop Science, 3(1): 8-13.

Ismail G., Sumerli M., Bicer B. T. and Yilmaz Y. (2005) . Heritability and correlation studies in pea (Pisum sativum L.) lines . Asian Journal of Plant Science , 4(2): 154-158.

Jinks J. L. and Hayman B. I. (1953). The analysis of diallel crosses . Maize Genetic Cooperation Newsletter 27:48-54.

Kumar D., Verma D. K and Singh N. K. (1997) .Heritability and expected genetic advance in pea (Pisum sativum L.) . Journal of Soils and Crops , 7(2):113-118.

Kumar H. and Das K. (1974) . Diallel analysis of yield and its components in pea. Indian Journal of Genetics and Plant Breeding , 34 (3) : 318-322.

Lila B., Sharma V. K., Chandra R.H. and Maurya S. K. (2009) . Studies on hybrid breeding and genetic variability in vegetable pea under high hilly condition of Uttarakhand. Annals of Horticulture , 2(2): 108-113.

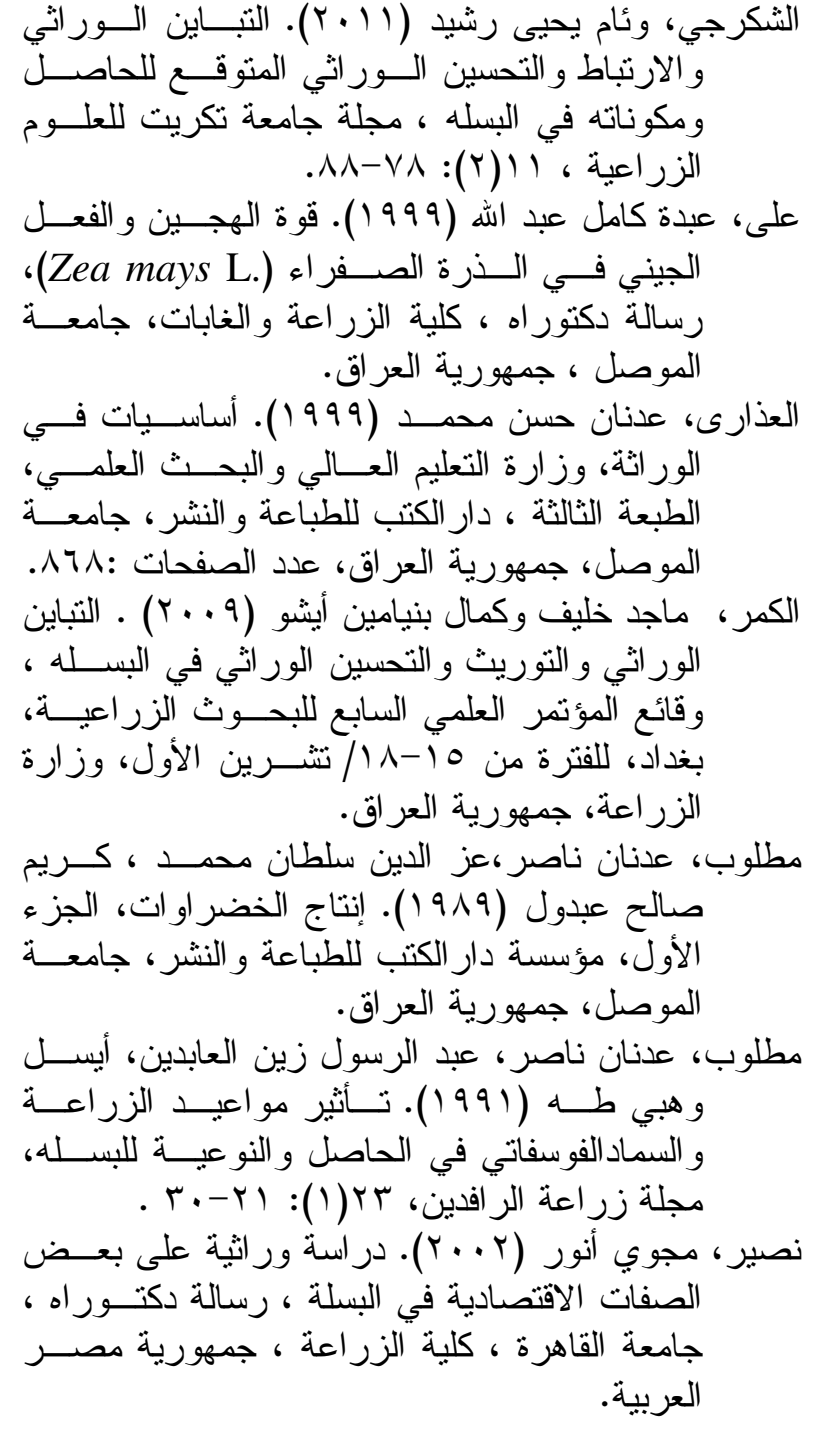

\section{REFERENCES}

Akansha S., Shalinia S. and Babu J. D. P. (2011). Heritability characters association and path analysis studies in early segregating population of field pea (Pisum sativum L. var. arvenses ) .International Journal of Plant Breeding and Genetic 5(1): 86- 92.

Ceyhan E. (2006). Combining abilities for grain yield and leaf characters in pea parents and crosses . Ziraat Fakultesi Derdisi, 20 (40): 83-89.

Chadha S., Sharma R.Chaudhury D. R. and Vidyassager V. (2008). Genetic variability studies in summer pea under cold desert areas of North-Westen Himalayas. Agricultural Science Digest, 28(1): 27-31.

Cruz C. D. (2001). Programa genes, aplicativo computacional em genetica e estatistica vicosa , Brazil.

Esmail R. M. (2007). Genetic analysis of yield 
Mather K. and Jinks J. L. (1982). Biometrical Genetics . Second Edn. Chapman and Hall Limited, London.

Murray L. W., Ray I. M ., Dong H. and Segovia A. L. (2003). The gardener and eberhart analysis 11 an 111 revisited. Crop Sci. 43: 1930- 1937.

Saieed N. T. (1990). Studies of variation in primary productivity morphology in velation to elective improvement of broadleaved tree species.Ph. D. Thesis, National University , Ireland.

Sardana S., Mahajan R.K., Gautam N. K. and Ram B. (2007). Genetic variability in pea (Pisum sativum L. ) germplasm for utilization . Sabrao Journal of Breeding and
Genetics, 39(1):31-42.

SAS (1985). Statistical analysis system . SAS Institute Inc. Cry , N.C. , U.SA.

Singh R. K. and Chaudhary B. D. (1985). Biometrical methods in quantitative genetics analysis. Rev. ed. Kalyani ,Publishers Ludhiana, India , P: 318.

Sood M. and Kalia P. (2006). Gene action of yield related traits in garden pea (Pisum sativum L.). Sabra Journal of Breeding and Genetics, 38(1): 1-17.

Viana J. M. S., Cruz C. D. and Cardoso A. A. (2001). Theory and analysis of partial diallel crosses, parents and F2 generations . Acta Sci., 23: 627- 634. 